\title{
Efeito do substrato sobre o crescimento de mudas de Mimosa bimucronata inoculadas com estirpes de rizóbio
}

\author{
Juliana Müller Freire ${ }^{1}$, Ederson da Conceição Jesus ${ }^{1}$, Janaína Ribeiro Costa Rouws ${ }^{1}$, Sérgio Miana da Faria ${ }^{1}$, Jerri Edson Zilli ${ }^{1}$ \\ ${ }^{1}$ Embrapa Agrobiologia, Rod. BR-465, Km 7, Bairro Ecologia, C P 74505, CEP 23891-000, Seropédica, RJ, Brasil
}

"Autor correspondente:

juliana.muller@embrapa.br

Termos para indexação:

Rizobactéria

Fixação de nitrogênio

Leguminosas arbóreas

Index terms:

Rhizobacteria

Nitrogen fixation

Leguminous treess

Histórico do artigo:

Recebido em 08/09/2015

Aprovado em 16/09/2016

Publicado em 30/06/2017

doi: 10.4336/2017.pfb.37.90.1132
Resumo - O objetivo deste trabalho foi avaliar a resposta de mudas de Mimosa bimucronata (DC) $\mathrm{O}$. Kuntze à inoculação com estirpes de rizóbio previamente selecionadas, utilizando diferentes substratos em condições de viveiro. Utilizou-se o delineamento experimental em blocos ao acaso com parcelas subdivididas, testando-se três substratos (organomineral puro contendo barro, areia e esterco na proporção 1:1:1 v:v:v, organomineral misturado com $30 \%$ de palha e areia com vermiculita na proporção 1:1 v:v) e quatro fontes de N (inoculação com as estirpes BR 3461 e BR 3470, tratamento controle com nitrogênio e controle sem adubação), totalizando 12 tratamentos. Foram avaliados altura e diâmetro do colo após 90 dias e massas da parte aérea, das raízes e dos nódulos secos após 120 dias em viveiro. Os substratos organominerais permitiram o melhor desenvolvimento das mudas. Entretanto, somente o substrato organomineral com palha e a areia com vermiculita permitiram resposta da planta à inoculação com a estirpe BR3470. O desempenho das mudas inoculadas não superou o das mudas adubadas com $\mathrm{N}$. A dose de $\mathrm{N}$ aplicada estimulou a nodulação ao invés de inibi-la.

\section{Effect of different substrates on growth of Mimosa bimucronata seedlings inoculate with rhizobium}

\begin{abstract}
The objective of this study was to evaluate the growth response of Mimosa bimucronata (DC) O. Kuntze seedlings in nursery conditions to inoculation with rhizobium strains previously selected using different substrates. An experimental design of randomized blocks with split plots was used, testing three substrates (pure organic-containing clay, sand and manure in $1: 1: 1 \mathrm{v}: \mathrm{v}: \mathrm{v}$ ratio; organomineral mixed with $30 \%$ straw and sand with vermiculite in $1: 1 \mathrm{v}: \mathrm{v}$ ) and four $\mathrm{N}$ sources (inoculation with strains BR 3461 and BR 3470, control with $\mathrm{N}$ fertilization and control without fertilization), totalizing 12 treatments. Height and stem diameter were evaluate after 90 days and shoot, root and nodules dry mass were evaluate after 120 days. Organomineral substrates provided better seedling growth. However, only organic-substrate with straw and sand with vermiculite showed positive responses of plants inoculated with BR3470 strain. The performance of the inoculated seedlings was not higher than that of seedlings fertilized with $\mathrm{N}$. The rate of $\mathrm{N}$ applied stimulated nodulation rather than inhibit it.
\end{abstract}

\section{Introdução}

A implementação do Plano ABC (agricultura de baixo carbono) (Brasil, 2017) e a aprovação do novo Código Florestal Brasileiro (Brasil, 2012), vem estabelecendo um cenário de aumento de demanda por reflorestamento.
Diversas metodologias de plantio têm sido adotadas para recuperação de áreas degradadas, dentre as quais se destaca a utilização de bactérias em simbiose com leguminosas. Essa tecnologia vem sendo pesquisada no Brasil há 25 anos, onde tem sido difundida e empregada com sucesso (Franco \& Faria, 1997; Chaer et al., 2011; 
Mendes et al., 2013; Ramos \& Souza, 2013). Entretanto, para que seja de fato efetiva, é fundamental que sejam selecionadas estirpes de rizóbios eficientes na nodulação e fixação do nitrogênio, pois muitas leguminosas arbóreas apresentam alta especificidade de nodulação (Faria \& Franco, 1997). Uma estirpe pode ser excelente fixadora para dada espécie leguminosa e ser incapaz de fixar N com outra (Faria \& Franco, 1997; Faria et al., 1999).

A comercialização de inoculantes é dependente da autorização pelo Ministério da Agricultura, Pecuária e Abastecimento, após passarem por um processo de validação que comprove sua eficiência agronômica em diferentes fases em laboratório e em campo. As estirpes atualmente autorizadas para venda constam na Instrução Normativa do Ministério de Agricultura, Pecuária e Abastecimento (MAPA) nº 13, de 24 de março de 2011 (Brasil, 2011). No bioma Mata Atlântica, das 377 espécies de leguminosas arbóreas nativas (Dutra \& Morim, 2015), 162 espécies são potencialmente noduladoras e 55 já tem algum registro de nodulação positivo confirmado pela literatura científica (Canosa et al., 2012). Entretanto, deste montante, apenas seis espécies têm inoculantes liberados para comercialização em função de normas de validação estabelecidas pelo MAPA. As espécies vegetais são: Balizia pedicellaris (DC.) Barneby \& J.W. Grimes, Dalbergia nigra (Vell.) Allemão ex Benth., Enterolobium contortisiliquum (Vell.) Morong, Erythrina verna Vell., Enterolobium timbouva Mart. e Pithecellobium tortum Mart.

Portanto, são necessárias pesquisas que comprovem a eficiência da inoculação para a grande maioria das espécies florestais (Umali-Garcia et al., 1988). Esse processo de validação possibilitará que os inoculantes sejam aprovados pelo MAPA para comercialização e disponibilizados para a sociedade. O maricá (Mimosa bimucronata) é uma espécie arbustiva fixadora, nativa dos biomas Caatinga, Cerrado e Mata Atlântica (Dutra \& Morim, 2015) e amplamente utilizada em projetos de restauração ambiental. Essa leguminosa tem reconhecida simbiose com rizóbios (Campêlo \& Campêlo, 1970; Lim, 1979), sendo os benefícios da inoculação destes microorganismos já comprovados na produção de suas mudas (Patreze \& Cordeiro, 2004). Entretanto, essa espécie foi retirada da lista de cepas/espécies autorizadas para venda, devido à falta de relatórios conclusivos sobre a eficiência da sua inoculação (Brasil, 2011).

Os substratos que contém compostos orgânicos são os mais utilizados em viveiros florestais para produção de mudas de espécies arbóreas (Xavier et al., 2009). Sabe-se que substratos ricos em nitrogênio podem inibir a nodulação de leguminosas e prejudicar a eficiência da inoculação de estirpes (Streeter, 1985; Tavares et al., 2016). Neste contexto, a avaliação da resposta à inoculação com rizóbios em substratos comumente utilizados em viveiros, com alternativas de imobilização do nitrogênio, é importante para adaptar as condições de produção ao uso destas tecnologias. A palha, por conter elevada relação $\mathrm{C} / \mathrm{N}$, tem o potencial de agir na imobilização do nitrogênio disponível na biomassa microbiana, favorecendo, assim, a retenção do $\mathrm{N}$ inorgânico liberado ao solo pelo processo de mineralização (Somasegaran \& Bohlool, 1990; Giacomini et al., 2009).

O objetivo deste trabalho foi avaliar o desempenho de mudas de maricá inoculadas com estirpes de rizóbio, utilizando diferentes tipos de substratos em condições não estéreis, em viveiro florestal.

\section{Material e métodos}

$\mathrm{O}$ experimento foi montado em viveiro comercial (Seropédica, RJ), utilizando as estirpes de rizóbio BR 3461 e BR 3470. O delineamento adotado foi o de blocos casualizados, com 5 repetições, em esquemas de parcelas subdivididas. Nas parcelas foram avaliados os substratos e nas subparcelas as fontes de N. O fator "substrato" foi divido em três níveis: (a) substrato organo-mineral sem palha; (b) substrato organo-mineral com palha; e (c) areia e vermiculita 1:1 (v:v). O fator "fonte de N" se dividiu em quatro níveis: (a) inoculação com a estirpe de rizóbio BR 3461; (b) inoculação com a estirpe de rizóbio BR 3470 ; (c) tratamento controle com adubação nitrogenada (adubação semanal com $8 \mathrm{mg}$ de nitrogênio na forma de $\mathrm{NH}_{4} \mathrm{NO}_{3}$, totalizando, ao final do experimento, $120 \mathrm{mg}$ de $\mathrm{N}$ aplicado por muda); e (d) tratamento controle sem adubação (ausência de inoculação e de adubação com N mineral). Em cada parcela foram utilizados 20 saquinhos de $1 \mathrm{~kg}$, totalizando 1.200 mudas no experimento. A distribuição dos tratamentos em parcelas subdivididas foi feita para evitar uma possível contaminação entre os tratamentos inoculados e os adubados com $\mathrm{N}$ mineral ou não adubados.

O substrato organo-mineral foi composto por areia, barro e esterco na proporção 1:1:1 (v:v:v). O barro foi obtido a partir do horizonte textural de um argissolo vermelho e amarelo, apresentando textura argilosa. No substrato organo-mineral com palha utilizou- 
se palha de capim napiê (Pennisetum purpureum Schum.), na proporção $30 \%$, incorporada ao substrato organomineral 60 dias antes do plantio, de modo a estimular a imobilização do $\mathrm{N}$ disponível e evitar que este influenciasse a nodulação das plantas. A análise química do substrato organo-mineral foi realizada antes de acrescentar a palha (Tabela 1), e não recebeu adubação extra, exceto o tratamento nitrogenado. A mistura de areia e vermiculita foi adubada com $4 \mathrm{~g} \mathrm{~kg}^{-1} \mathrm{de}$ superfosfato simples, $0,25 \mathrm{~g} \mathrm{~kg}^{-1}$ de sulfato de potássio e $0,25 \mathrm{~g} \mathrm{~kg}^{-1}$ de FTE-BR12 (Freire et al., 2013).

Tabela 1. Análise química dos substratos utilizados no experimento antes da aplicação da adubação química, da palha e dos inoculantes BR 3461 e BR 3470.

\begin{tabular}{|c|c|c|c|c|c|c|c|c|c|}
\hline Substrato & $\begin{array}{c}\mathrm{pH} \\
\text { (unid) }\end{array}$ & $\begin{array}{c}\mathrm{Al} \\
\left(\mathrm{cmol}_{\mathrm{c}} \mathrm{dm}^{-3}\right)\end{array}$ & $\begin{array}{c}\mathbf{K} \\
\left(\mathrm{mg} \mathrm{L}^{-1}\right)\end{array}$ & $\begin{array}{c}\mathrm{Ca} \\
\left(\mathrm{cmol}_{\mathrm{c}} \mathrm{dm}^{-3}\right)\end{array}$ & $\begin{array}{c}\mathrm{Mg} \\
\left(\mathrm{cmol}_{\mathrm{c}} \mathrm{dm}^{-3}\right)\end{array}$ & $\begin{array}{c}P \\
\left(m g L^{-1}\right)\end{array}$ & $\begin{array}{c}\mathrm{C} \\
(\%)\end{array}$ & $\begin{array}{c}\text { Acidez } \\
(\mathbf{H}+\mathbf{A l})\end{array}$ & $\begin{array}{c}N \\
(\%)\end{array}$ \\
\hline $\begin{array}{l}\text { Organo-mineral } \\
\text { (Areia/ argila/ } \\
\text { esterco) }\end{array}$ & 7,57 & 0 & 580 & 3,46 & 2,14 & 48,06 & 1,26 & 0,28 & 0,150 \\
\hline Areia/vermiculita & 7,44 & 0 & 128 & 0,98 & 17,20 & 1,90 & 0,25 & 0,61 & 0,001 \\
\hline
\end{tabular}

As estirpes BR 3461 e BR 3470 foram selecionadas em ensaios prévios no laboratório de leguminosas da Embrapa Agrobiologia e testadas em vasos Leonard e solo não estéril (Oliveira Júnior et al., 2010) conforme a recomendação do Ministério da Agricultura, Pecuária e Abastecimento (Franco \& Faria, 1997; Moreira et al., 2010; Brasil, 2011). Os inoculantes foram preparados no laboratório de bioprocessos da Embrapa Agrobiologia, utilizando como veículo a turfa (mínimo de $10^{8} \mathrm{UFC}^{-1}$ ).

As sementes de maricá (Mimosa bimucronata) foram escarificadas com ácido sulfúrico por $5 \mathrm{~min}$, utilizando recomendação de Ribas et al. (1996). As sementes foram inoculadas utilizando-se goma de amido (3\%), permitindo maior aderência às sementes (Fernandes Júnior et al., 2012). Posteriormente, foram colocadas para secar à sombra e semeadas a $2 \mathrm{~cm}$ de profundidade em sacos plásticos contendo $1 \mathrm{~kg}$ dos diferentes substratos.

O experimento foi implantado em novembro de 2013. Aos 90 dias foram analisadas as variáveis: altura (h), diâmetro à altura do colo (DAC). Aos 120 dias o experimento foi desmontado e medidas as seguintes variáveis: massa de nódulos secos (MNS), massa de parte aérea seca (MPAS), massa de raízes secas (MRS) e massa seca total (MST). A altura e o DAC foram analisados para 10 plantas aleatoriamente, e as massas secas para 7 plantas dentro de cada parcela. As raízes foram lavadas e secas em estufa de circulação de ar a $65{ }^{\circ} \mathrm{C}$ por $72 \mathrm{~h}$. Posteriormente, os nódulos foram retirados manualmente, secos novamente em estufa e pesados.
Todos os dados obtidos foram submetidos à análise de variância e ao teste de Scott-Knott a 5\% de probabilidade, para comparação das médias. Os resultados obtidos das massas de parte aérea, raízes e nódulos secos foram transformados em log antes de serem analisados.

\section{Resultados e discussão}

De maneira geral, os substratos organominerais foram os que promoveram maior incremento em altura (h), diâmetro à altura do colo (DAC), massa seca da parte aérea (MPAS), raízes (MRS), total (MTS) e de nódulos secos (MNS). Dentre as fontes de N, a que promoveu os maiores valores em todas as variáveis, incluindo a massa dos nódulos, foi o N mineral (Figuras 1a-d; Figura 2). A presença de maior quantidade de nutrientes no solo nos substratos organominerais em relação ao substrato areia com vermiculita (Tabela 1) foi, provavelmente, o fator responsável pelo melhor crescimento das mudas em relação às variáveis analisadas.

A interação entre os fatores fontes $\mathrm{N}$ x substrato foi significativa ao nível de $5 \%$ de probabilidade para as variáveis altura, MPAS, MRS e MTS (Tabela 2). Nestes casos, dependendo da variável e do substrato utilizado houve uma resposta diferenciada da fonte de $\mathrm{N}$, conforme pode ser visualizado na Figura 1. O mesmo ocorreu quando se comparou os substratos considerando uma mesma fonte de $\mathrm{N}$. 
Tabela 2. Resumo da análise de variância do crescimento de mudas de maricá (Mimosa bimucronata) cultivadas em diferentes substratos e submetidas a diferentes fontes de nitrogênio.

\begin{tabular}{cccccccc}
\hline & \multicolumn{7}{c}{ Quadrado Médio } \\
\cline { 2 - 7 } $\begin{array}{c}\text { Fonte de } \\
\text { variação }\end{array}$ & GL & Altura & DAC & MPAS & MRS & MTS & MNS \\
\hline Bloco & 4 & $121,170^{\text {ns }}$ & $0,5176^{\text {ns }}$ & $0,0147^{\text {ns }}$ & $0,0206^{*}$ & $0,0261^{\text {ns }}$ & $0,0031^{\text {ns }}$ \\
Fontes N & 3 & $2128,83^{* *}$ & $14,934^{* *}$ & $0,8309^{* *}$ & $0,3167^{* *}$ & $0,8335^{* *}$ & $0,0129^{* *}$ \\
Erro (a) & 12 & 65,41 & 0,185 & 0,0138 & 0,0046 & 0,0108 & 0,0022 \\
Substrato & 2 & $2915,45^{* *}$ & $16,804^{* *}$ & $1,1161^{* *}$ & $0,7850^{* *}$ & $1,5597^{* *}$ & $0,0331^{* *}$ \\
Fontes N x & 6 & $172,08^{* *}$ & $0,3513^{\text {ns }}$ & $0,0545^{* *}$ & $0,0506^{* *}$ & $0,0946^{* *}$ & $0,0021^{\text {ns }}$ \\
substrato & & & & & & & \\
Erro (b) & 32 & 33,97 & 0,1758 & 0,0101 & 0,0044 & 0,0090 & 0,0016 \\
CVa (\%) & & 15,74 & 12,79 & 18,67 & 14,45 & 13,38 & 62,47 \\
CVb (\%) & & 11,34 & 12,46 & 15,94 & 14,14 & 12,21 & 53,69 \\
\hline
\end{tabular}

** e * Efeito significativo pelo teste Scott-Knott ao nível de 1\% e $5 \%$ de probabilidade, respectivamente; ns - não significativo. CV: coeficiente de variação. DAC = diâmetro à altura do colo; MPAS, MRS, MTS e MNS = massa seca da parte aérea, das raízes, total e dos nódulos, respectivamente.

Interpretando a interação significativa entre fontes de $\mathrm{N}$ e substrato, considerando a variável altura, podemos observar na Figura 1 que os maiores valores de altura das mudas inoculadas com BR 3461, controles com adubação nitrogenada ou sem adubação ocorreram nos substratos organominerais (com ou sem palha). Para a estirpe BR 3470 o substrato estatisticamente superior foi o orgânico sem palha, superando significativamente a altura proporcionada pelo substrato orgânico com palha, que por sua vez superou o substrato areia com vermiculita.

Para a variável MPAS, os maiores valores médios das mudas inoculadas com a estirpe BR 3470, adubação nitrogenada e controle sem adubação, ocorreram nos substratos organominerais, com exceção da estirpe BR 3461 que foi estatisticamente superior no substrato orgânico sem palha em relação ao substrato orgânico com palha e areia com vermiculita, que se igualaram. Comportamento semelhante foi observado para a variável MTS, que foi maior no substrato orgânico sem palha, estatisticamente superior ao substrato orgânico com palha e este por sua vez foi maior do que a areia com vermiculita.

Também para MRS, os substratos organominerais foram superiores para a maioria das fontes de $\mathrm{N}$, com exceção quando se utilizou a estirpe BR 3470, que resultou em mudas com valor médio de MRS significativamente superior no substrato orgânico com palha, e inferior na areia com vermiculita.
Para as variáveis diâmetro à altura do colo (DAC) e massa de nódulos secos (MNS), a interação entre fontes $\mathrm{N}$ x substrato não foi significativa (Tabela 1). Ou seja, independente da fonte de $\mathrm{N}$ testada, a MNS e o DAC foram significativamente maiores nos substratos organominerais e menores na areia com vermiculita. A adubação nitrogenada foi estatisticamente superior às mudas inoculadas e ao tratamento sem adubação em relação ao DAC e a MNS, mas não deferiram entre si. Nestes casos, portanto, somente os fatores substrato e a fonte de N, isolados, foram significativos (Figura 2).

Os controles absolutos também apresentaram nódulos, indicando a presença de populações de bactérias nativas capazes de nodular Mimosa bimucronata no solo. Esperava-se que a massa de nódulos secos fosse menor nas mudas produzidas sob substrato organomineral, devido à presença de maior quantidade nitrogênio no solo, o que supostamente inibiria a nodulação. Entretanto, esta inibição não ocorreu, e justamente as plantas do tratamento controle com adubação nitrogenada, independente do tipo de solo, foram as que apresentaram os maiores valores de MNS. A menor quantidade de nódulos secos no substrato areia com vermiculita pode ter sido influenciada pela baixa fertilidade natural deste substrato. A deficiência nutricional do solo pode afetar a nodulação e a fixação biológica de nitrogênio (Bonilla \& Bolaños, 2009). 

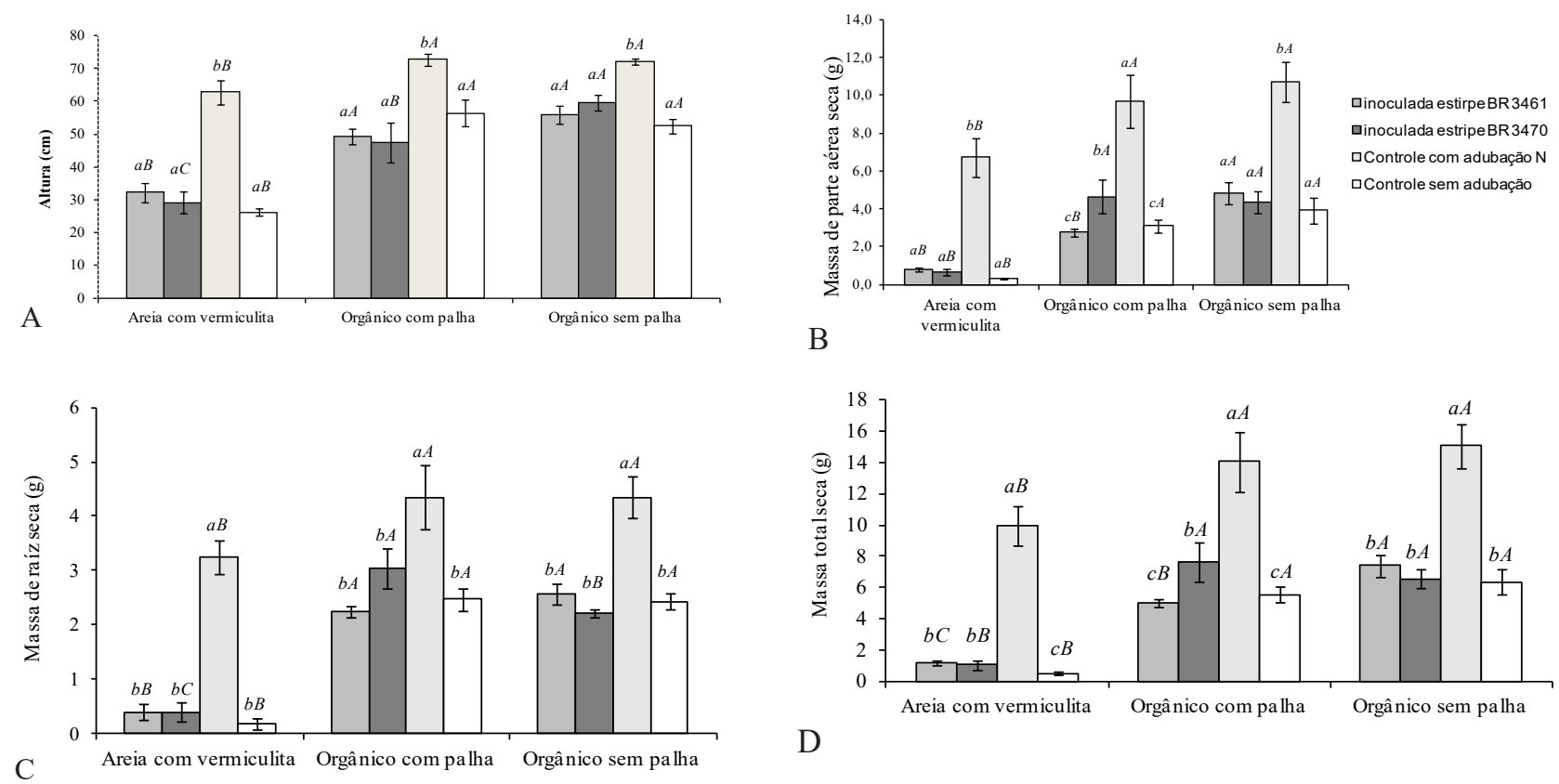

Figura 1a-d. A) Altura, massa seca de b) parte aérea, c) de raízes e d) total de mudas de maricá (Mimosa bimucronata), em diferentes substratos e fontes de $\mathrm{N}$ e substratos (com interação significativa entre estes fatores). Barras seguidas de letras distintas, minúsculas entre fontes de $\mathrm{N}$ e maiúsculas entre substratos para uma mesma fonte de $\mathrm{N}$, diferem entre si pelo teste de Scott Knott, a 5\% de probabilidade.
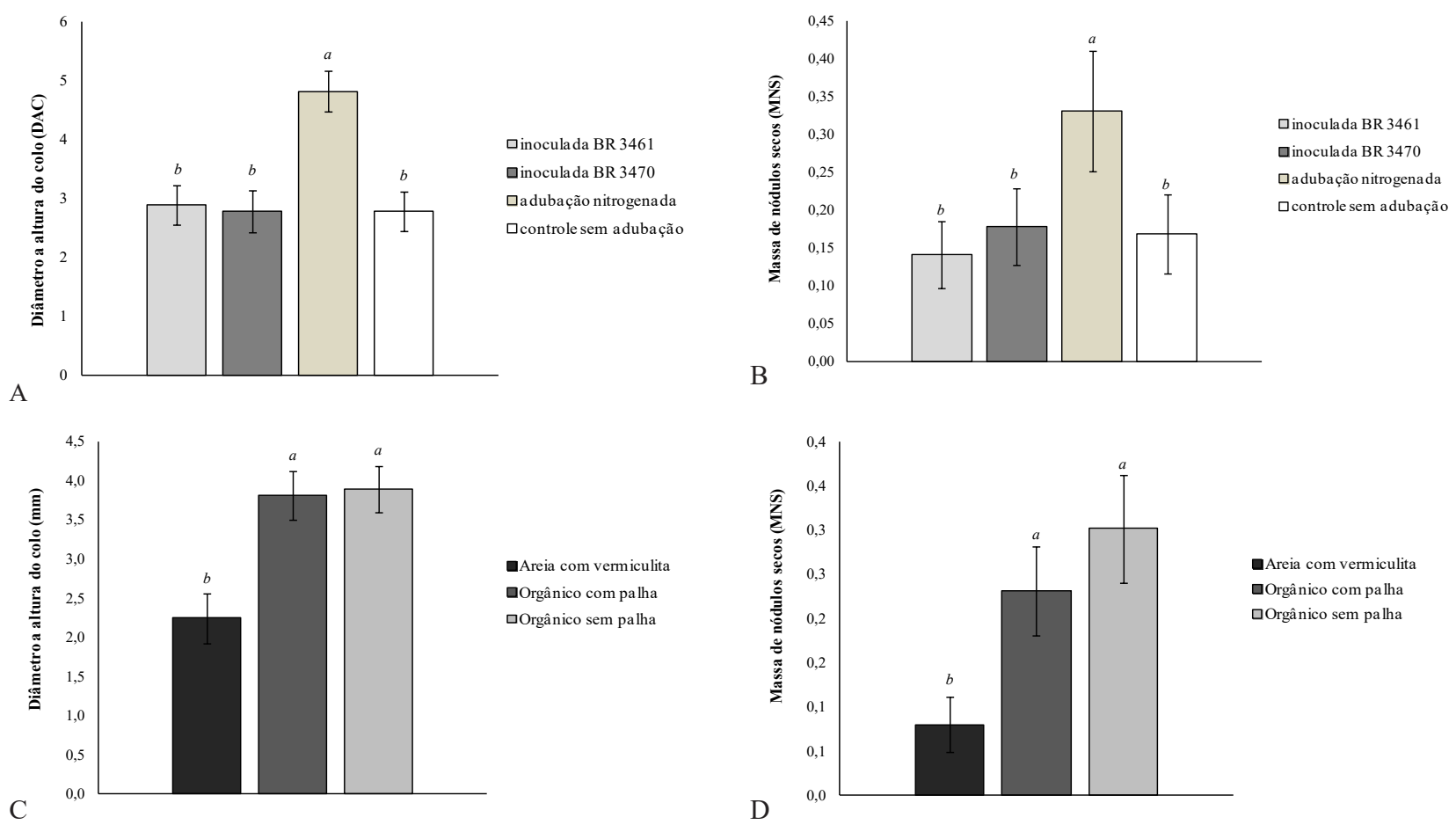

Figura 2 a-d. Médias gerais de a e b) massa de nódulos secos e c e d) diâmetro a altura do colo em diferentes substratos e fontes de $\mathrm{N}$ (sem interação significativa entre estes fatores). Barras seguidas de letras distintas diferem entre si pelo teste de Scott Knott, a 5\% de probabilidade. 
Amaior média de massa de nódulos secos no tratamento nitrogenado indica que a adubação nitrogenada na dose utilizada neste experimento, de $120 \mathrm{mg}$ de $\mathrm{N}$ por muda, que corresponde a $60 \mathrm{~kg} \mathrm{~N} \mathrm{ha}^{-1}$, não foi suficiente para inibir a nodulação com rizóbios naturalmente presentes no substrato, pelo contrário, estimulou a simbiose (Fig. 2A). Sabe-se que o $\mathrm{N}$ quando aplicado em pequenas doses pode beneficiar a nodulação (Ingestad, 1980; Gonçalves et al., 1999; Jesus et al., 2014). Porém, quando aplicado em doses mais altas, o $\mathrm{N}$ inibe a nodulação de espécies arbóreas (Umali-Garcia et al., 1988). Patreze \& Cordeiro (2004) também observaram a presença de nódulos em mudas de $M$. bimucronata adubadas com $\mathrm{N}$ na forma de nitrato de amônio, em doses de $640 \mathrm{mg}$ ou $320 \mathrm{~kg} \mathrm{~N}$ ha $^{-1}$, bastante superior ao utilizado no presente estudo.

Stamford \& Silva (2000) observaram a nodulação de $M$. caesalpiniifolia por rizóbios nativos, mesmo recebendo adubação com $\mathrm{N}$ mineral na dose de $100 \mathrm{~kg}$ $\mathrm{ha}^{-1}$. Resultados similares também foram observados por Cruz et al. (1997) com aplicação de $200 \mathrm{~kg} \mathrm{ha}^{-1} \mathrm{~N}$ em jacatupé (Pachyrhizus erosus) e por Chen et al. (1992) em soja.

O crescimento das mudas inoculadas, de maneira geral, foi muito menor que das mudas que receberam adubação mineral de $\mathrm{N}$. Este resultado pode ter relação com a baixa competitividade destas estirpes em condições não esterilizadas, e pelo fato das mudas adubadas com $\mathrm{N}$ mineral terem nodulado. Resultado semelhante foi encontrado por Góes et al. (2015) na produção de mudas inoculadas de Inga laurina (SW.) Willd.

As mudas inoculadas apresentaram MPAS e MTS estatisticamente maiores que das mudas do tratamento controle sem adubação. Isso ocorreu nos substratos organo-mineral com palha e areia com vermiculita (Figuras 1b e 1d), mas não no substrato organo-mineral sem palha, para o qual não houve diferença entre os tratamentos inoculados e o tratamento controle sem adubação para nenhuma variável. No substrato organomineral com palha, a muda inoculada com a estirpe BR 3470 apresentou MPAS de 4,643 g, o controle sem adubação 3,098 g e a muda inoculada com a estirpe BR 3461, 2,758 g. Entretanto este valor ainda foi bem inferior ao da muda adubada com $\mathrm{N}$, que apresentou 9,693 g (Figura. 1b). Ainda neste substrato, a muda inoculada com a BR 3470 apresentou MTS de 7,68 g, significativamente maior que a inoculada com a BR 3461 $(5,01 \mathrm{~g})$ e o controle sem adubação $(5,58 \mathrm{~g})$, mas ainda menor do que a muda adubada com N (14,04 g), como pode ser observado na Figura 1d.

No substrato areia com vermiculita, as mudas inoculadas, independente da estirpe, também apresentaram valores de MTS estatisticamente maiores que o controle sem adubação (Figura 1d). Desta maneira, alguma resposta da espécie à inoculação pode ser observada com o uso do substrato organomineral com palha e a areia com vermiculita. A baixa quantidade de N, no caso da areia com vermiculita (Tabela1), ou a sua imobilização pela palha, no caso do substrato organomineral com palha, podem ter favorecido a nodulação pelas bactérias ou reduzido a competitividade das bactérias inoculadas com aquelas presentes no solo.

A eficiência da inoculação foi observada por Vieira \& Souza (2011) para mudas de Swartzia argentea após 204 dias em viveiro para as variáveis massa do caule seco e MNS e para S. laevicarpa após 185 para as variáveis DAC, MPAS, MRS e caule secos. De forma semelhante ao encontrado no presente estudo, as variáveis que apontaram maior eficiência da inoculação para Swartzia sp, com exceção da MNS, não diferiram entre o tratamento inoculado e o tratamento nitrogenado, mas somente entre o tratamento inoculado e o controle sem adubação. Almeida et al. (2013) também observaram resposta positiva de Inga edulis à inoculação com bactérias diazotróficas, com a produção de mudas de melhor qualidade e maior crescimento em altura, DAC e MTS, desta vez com diferenciação em relação aos tratamentos controle com e sem adubação.

Tavares et al. (2016), estudando a inoculação com rizóbio e micorrizas em mudas de Acacia mangium Willd produzidas em dois tipos de substratos com diferentes teores de matéria orgânica, observaram menor quantidade de MNS no substrato com maior teor de matéria orgânica, submetido à dupla inoculação. Moreira et al. (1996) avaliaram a produção de mudas de Swartzia laevicarpa Amshoff. em cinco substratos (areia, convencional, solo de várzea, terriço de floresta com adubo e sem adubo) em condições de viveiro e encontraram melhor resultado de nodulação e dos demais parâmetros da muda no substrato convencional composto apenas por mistura de argila e areia na proporção $3: 2$ (v:v). 


\section{Conclusões}

A melhor resposta à inoculação de Mimosa bimucronata em condição de viveiro foi observada com a estirpe BR3470, utilizando-se substrato organomineral com palha.

Os substratos organominerais permitem o melhor desenvolvimento das mudas de Mimosa bimucronata, entretanto, somente os substrato com palha e com areia e vermiculita proporcionam resposta das mudas à inoculação com estirpes de rizóbio BR3470.

A adubação nitrogenada promoveu o maior desenvolvimento das mudas, sendo superior às mudas inoculadas com estirpes de rizóbios, para todas as variáveis analisadas. A dose de nitrogênio utilizada (de $120 \mathrm{mg} \mathrm{N} \mathrm{muda}^{-1}$ ) estimulou a nodulação de Mimosa bimucronata ao invés de inibi-la.

\section{Referências}

Almeida, G. S. et al. Capacidade de nodulação em Inga sp. de ocorrência na Amazônia Ocidental. Enciclopédia Biosfera, v. 9, p. 491-508, 2013.

Bonilla, I. \& Bolaños, L. Mineral nutrition for legume-rhizobia symbiosis: $\mathrm{B}, \mathrm{Ca}, \mathrm{N}, \mathrm{P}, \mathrm{S}, \mathrm{K}, \mathrm{Fe}, \mathrm{Mo}, \mathrm{Co}$, and $\mathrm{Ni}$ : A review. In: Lichtfouse, E. (Ed.). Organic farming, pest control and remediation of soil pollutants. Netherlands: Springer, 2009. p. 253-274. DOI: 10.1007/978-1-4020-9654-9.

BRASIL. Lei $\mathrm{n}^{\circ} 12.651$, de 25 de maio de 2012. Dispõe sobre a proteção da vegetação nativa [...] e dá outras providências. Diário Oficial [da] República Federativa do Brasil, n. 102, 28 maio, 2012. Disponível em: <http://www.planalto.gov.br/ccivil_03/_ato20112014/2012/lei/L12651 compilado.htm>. Acesso em: 23 set. 2016.

Brasil. Ministério da Agricultura, Pecuária e Abastecimento. Instrução Normativa $\mathrm{n}^{\mathrm{o}}$ 13, de 24 de março de 2011. Diário Oficial [da] República Federativa do Brasil, Brasília, DF, n. 58, p. 3, 25 mar. 2011.

Brasil. Ministério da Agricultura, Pecuária e Abastecimento. Plano ABC. Brasília, DF, 2017. Disponível em: $<$ http://www.agricultura. gov.br/assuntos/sustentabilidade/plano-abc $>$. Acesso em: 23 set. 2016.

Campêlo, A. B. \& Campêlo, C. R. Eficiência da inoculação cruzada entre espécies da subfamília Mimosoideae. Pesquisa Agropecuária Brasileira, v. 5, n. 1, p. 333-337, 1970.

Canosa, G. A. et al. Leguminosas florestais fixadoras de nitrogênio da Mata Atlântica brasileira para uso em sistemas agroflorestais e na restauração ecológica. Seropédica: Embrapa Agrobiologia, 2012. (Embrapa Agrobiologia. Comunicado técnico, 144).

Chaer, G. M. et al. Nitrogen-fixing legume tree species for the reclamation of severely degraded lands in Brazil. Tree Physiology, v. 31, p. 139-149, 2011. DOI: 10.1093/treephys/tpq116.
Chen, Z. et al. Soybean nodulation and grain yield as influenced by $\mathrm{N}$ : fertilizer rate, plant population of city and cultivar in Southern Quebec. Canadian Journal of Plant Science, v. 72, n. 4, p. 10491056, 1992. DOI: 10.4141/cjps92-131.

Cruz, G. N. et al. Effects of inoculation with Bradyrhizobium and urea application on nitrogen fixation and growth of yam bean (Pachyrhizus erosus) as affected by phosphorus fertilizers in an acid soil. Tropical Grasslands, v. 31, p. 538-542, 1997.

Dutra, V. F. \& Morim, M. P. Mimosa in lista de espécies da flora do Brasil. Rio de Janeiro: Instituto Jardim Botânico do Rio de Janeiro, 2015. Disponível em: < http://floradobrasil.jbrj.gov.br/jabot/ floradobrasil/>. Acesso em: 30 abr. 2015.

Faria, S. M. de \& Franco, A. A. Obtenção de inoculantes eficientes para fixação biológica de nitrogênio em espécies leguminosas arbóreas. Seropédica: EMBRAPA-CNPAB, 1997. (EMBRAPACNPAB. Recomendação técnica, 153).

Faria, S. M. de et al. Nodulação em espécies florestais, especificidade hospedeira e implicações na sistemática de leguminosae. In: Siqueira, J. O. et al. (Ed.). Inter-Relação fertilidade, biologia do solo e nutrição de plantas. Viçosa, MG: SBCS; Lavras: UFLA/DCS, 1999. p. 667-686.

Fernandes Júnior, P. I. F. et al. Performance of polymer compositions as carrier to cowpea rhizobial inoculant formulations: survival of rhizobia in pre-inoculated seeds and field efficiency. African Journal of Biotechnology, v. 11, p. 2945-2951, 2012. DOI: 10.5897/ AJB11.1885.

Franco, A. A. \& Faria, S. M. de. The contribution of N2-fixing tree legumes to land reclamation and sustainability in the tropics. Soil Biology and Biochemistry, v. 29, n. 5/6, p. 897-903, 1997. DOI: 10.1016/S0038-0717(96)00229-5.

Freire, L. R. et al. (Org.). Manual de calagem e adubação do Estado do Rio de Janeiro. Brasília, DF: Embrapa, 2013. 430 p.

Giacomini, S. J. et al. Imobilização do nitrogênio amoniacal de dejetos líquidos de suínos em plantio direto e preparo reduzido do solo. Revista Brasileira de Ciência do Solo, v. 33, p. 41-50, 2009. DOI: 10.1590/S010006832009000100005.

Góes, G. S. et al. Efeitos da inoculação com bactérias diazotróficas e da adubação nitrogenada no crescimento e na qualidade de mudas de Inga laurina (Sw.) Willd. (fabaceae). Revista Árvore, v. 39, n. 6, p. 1031-1038, 2015. DOI: 10.1590/0100-67622015000600005.

Gonçalves, C. A. et al. Crescimento e nodulação de Inga marginata em resposta à adição de nitrogênio, fósforo e inoculação com rizóbio. Floresta e Ambiente, v. 6, n. 1, p. 118-126, 1999.

Ingestad, T. Grownt, nutrition and nitrogen fixation in grey alder at variety rate of nitrogen fixation addition. Physiologia Plantarum, n. 50, p. 353-364, 1980. DOI: 10.1111/j.1399-3054.1980.tb04113.x.

Jesus, A. A. et al. Qualidade de mudas de Enterolobium contortisiliquum (Vell.) Morong. em função da inoculação e nodulação natural em solos do Sudoeste Piauiense, Brasil. Revista de Ciências Agrárias, v. 37, n. 2, p. 198-205, 2014.

Lim, G. Rhizobium and nodulation of legumes in Singapore. In: Broughton, W. J. et al. (Ed.). Soil microbiology and plant nutrition. Kuala Lumpur: University of Malaya, 1979. p. 159-175. 
Mendes, M. M. C. et al. Crescimento e sobrevivência de mudas de sabiá (Mimosa caesalpiniaefolia Benth.) inoculadas com microorganismos simbiontes em condições de campo. Ciência Florestal, v. 23, n. 2, p. 309-320, 2013. DOI: 10.5902/198050989277.

Moreira, F. M. S. et al. Bactérias fixadoras de N2 e fungos micorrízicos arbusculares em espécies florestais: avanços e aplicações biotenológicas. In: Figueiredo, M. do V. B. et al. (Ed.). Biotecnologia aplicada à agricultura: textos de apoio e protocolos experimentais. Brasília, DF: Embrapa Informação Tecnológica; Recife: Instituto Agronômico de Pernambuco, 2010. p. 439-477.

Moreira, F. W. et al. Germinação, crescimento inicial e nodulação em viveiro de saboarana (Swartzia laevicarpa Amshoff). Acta Amazônica, v. 25, n. 3/4, p. 149-160, 1995. DOI: 10.1590/180943921995253160.

Oliveira Júnior, J. Q. de et al. Estirpes de rizóbio indicadas para a inoculação de espécies de leguminosas florestais: aproximação 2010. Seropédica: Embrapa Agrobiologia, 2010. (Embrapa Agrobiologia. Documentos, 268).

Patreze, C. M. \& Cordeiro, L. Nitrogen-fixing and vesiculararbuscular mycorrhizal symbioses in some tropical legume trees of tribe Mimoseae. Forest Ecology and Management, v. 196, p. 275-285, 2004. DOI: 10.1016/j.foreco.2004.03.034.

Ramos, D. B. P. \& Souza, L. A. G. Seleção de estirpes de rizóbios para formação de mudas de leucena (Leucaena leucocephala (Lam) De Wit.) em Argissolo Vermelho Amarelo. Revista Brasileira de Agroecologia, v. 8, n. 1, 2013.

Ribas, L. L. F. et al. Superação de dormência de sementes de Mimosa bimucronata (DC.) O. Kuntze (maricá). Revista Brasileira de Sementes, v. 18, n. 1, p. 98-101, 1996.
Somasegaran, P. \& Bohlool, B. B. Single-strain versus multistrain inoculation: effect of soil mineral $\mathrm{n}$ availability on rhizobial strain effectiveness and competition for nodulation on chick-pea, soybean, and dry beant. Applied and environmental Microbiology, v. 56, n. 11, p. 3298-3303, 1990. DOI: 0099-2240/90/113298-06\$02.00/0.

Stamford, N. P. \& Silva, R. A. da. Efeito da calagem e inoculação de sabiá em solo da mata úmida e do semi-árido de Pernambuco. Pesquisa Agropecuária Brasileira, v. 35, n. 5, p. 1037-1045, 2000. DOI: 10.1590/S0100-204X2000000500023.

Streeter, J. G. Nitrate inhibition of legume nodule growth and activity. I. Long-term studies of a continuous supply of nitrate. Plant Physiology, n.77, p. 321-324, 1985. DOI: 00320889/85/77/0325/04/\$0 1.00/0

Tavares, S. R. de L. et al. Produção de mudas de Acacia mangium Willd noduladas e micorrizadas em diferentes substratos. HOLOS, v. 4, p. 56-66, 2016. DOI: 10.15628/holos.2016.3931.

Umali-Garcia, M. et al. Effects of Rhizobium inoculation on growth and nodulation of Albizia falcataria (L.) Fosh. and Acacia mangium Willd. in the nursery. Plant and Soil, v. 108, n. 1, p. 71-78, 1988. DOI: $10.1007 / B F 02370101$.

Vieira, E. P. \& Souza, L. Inoculação com rizóbios em mudas de acapu do igapó e saboarana. Amazonian Journal of Agricultural and Environmental Sciences, v. 54, n. 1, p. 54-62, 2011.

Xavier, A. et al. Silvicultura clonal: princípios e técnicas. Viçosa, MG: Ed. UFV, 2009. 272 p. 